\title{
ADAPTIVE BLIND MULTIUSER DETECTION UNDER IMPULSIVE NOISE USING PRINCIPAL COMPONENTS
}

\author{
R. Nirmala Devi ${ }^{1}$ and K.Kishan Rao ${ }^{2}$ \\ ${ }^{1}$ Asooc .Prof., Department of Elelctronics and Instrumentation, Kakatiya \\ Institute of Technology And Science Warangal, Andhrapradesh, India \\ ${ }^{2}$ Director Vagdevi Group of Institutions, Bollikunta Warangal, Andhrapradesh, \\ India
}

\begin{abstract}
In this paper we consider blind signal detection for an asynchronous code division multiple access (CDMA) system with Principal component analysis (PCA) in impulsive noise. The blind multiuser detector requires no training sequences compared with the conventional multiuser detection receiver. The proposed PCA blind multiuser detector is robust when compared with knowledge based signature waveforms and the timing of the user of interest. PCA is a statistical method for reducing the dimension of data set, spectral decomposition of the covariance matrix of the dataset i.e first and second order statistics are estimated. Principal component analysis makes no assumption on the independence of the data vectors PCA searches for linear combinations with the largest variances and when several linear combinations are needed, it considers variances in decreasing order of importance. PCA improves SNR of signals used for differential side channel analysis. In different to other approaches, the linear minimum mean-square-error (MMSE) detector is obtained blindly; the detector does not use any training sequence like in subspace methods to detect multi user receiver. The algorithm need not estimate the subspace rank in order to reduce the computational complexity. Simulation results show that the new algorithm offers substantial performance gains over the traditional subspace methods.
\end{abstract}

\section{KEYWORDS}

Blind Multiuser Detection, Code Division Multiple Access, Inter Symbol Inter-ference (ISI). Multiple Access Interference (MAI). Principal component analysis (PCA)

\section{INTRODUCTION}

In DS-CDMA detector uses one user detection strategy in which each user is taken as a signal, while the other users are considered as noise. In recent years, multiuser detection has attracted significant interest due to enhancement of performance it offers in communication schemes with present multiple access interference (MAI) [1]. Blind multiuser detection is effective for reducing inter symbol interference (ISI) and improving system throughput. Among all blind methods the

Sundarapandian et al. (Eds) : ACITY, AIAA, CNSA, DPPR, NeCoM, WeST, DMS, P2PTM, VLSI - 2013 pp. 123-131, 2013. () CS \& IT-CSCP 2013

DOI : $10.5121 /$ csit.2013.3413 
subspace method (SS) [2] is known for its better performance. However, the batch algorithm [2] requires singular value decomposition (SVD), which is inconvenient for adaptive implementation. Further, it requires accurate rank estimation of the correlation matrix, which is not easy in an inherently noisy environment [4]-[9].

All the subbspace tracking algorithms were found with computational complexity around $O\left(\mathrm{~m}^{2}\right)$, or $O(m d)$ where $\mathrm{d}$ is the dimension of the signal subspace, in each recursion [3]. However, the total computation of the blind channel identification is still $O\left(\mathrm{~m}^{3}\right)$ due to the second step, i.e., using the estimated subspace vectors to optimize channel estimation. The second step cannot be repeatedly implemented with reduced computations in an obvious manner[11]. Least square(LS) method is a simple way to estimate a Channel ,it's efficiency is not significant . Principal component analysis does not assume on the independence of the data vectors. When many combinations are required PCA identifies linear combinations with the largest variances, it considers variances in decreasing order of importance. In PCA signal to noise ratio of signals used for differential side channel analysis is improved. By applying PCA to the frequency spectrum noise and signals can be separated.

These detectors employ the PCA algorithm [9] at the outputs of a bank of matched filters. Main purpose is to retrive the original sequence from the received signal that is corrupted by other users and MAI, without the help of training sequences and a priori knowledge of the channel.

The paper is organized as follows :Section 2 describes the System model followed by section 3 with the description of the algorithm used. In section 4 contains results and conclusions.

\section{SYSTEM MODELLING}

Consider a DS-CDMA channel operating with coherent binary phase shift keying (BPSK) modulation format with $L$ users sharing the same bandwidth. If $L$ users are active, the received base band, continuous-time signal is a superposition of all $L$ signals[4],

$$
y(t)=X(t)+z(t),-\infty \leq t \leq \infty
$$

where $X(t)$ is the useful signal comprised of the data signals of $L$ active users and $z(t)$ the additive white Gaussian noise. The useful signal $X(t)$ is taken as the expression

$$
X(t)=\sum_{l=1}^{L} w_{l} \sum_{i=0}^{M-1} b_{l}(i) x_{l}\left(t-i T-\tau_{l}\right)
$$

where $M$ stands for the number of data symbols per user in the data frame of interest, $T$ is the symbol interval, $w_{l}$ is the received signal amplitude, $\tau_{l}$ is the delay, $\left\{b_{l}(i), \quad i=0,1, \ldots ., M-1\right\}$ is the symbol stream, and $x_{l}(t),\left\{x_{l}(t) ; 0 \leq t \leq T\right\}$ is the normalized signaling waveform of the $l$ th user. It is assumed that the signaling interval of each user is $T$ seconds, and the input alphabet is

antipodal binary: $[-1,+1]$. Further, direct-sequence spread-spectrum (DS-SS) multiple access signatures may be written as a succession of the chip pulses as 
$x_{l}(t)=\sum_{j=0}^{N-1} p_{j}^{l} \varphi\left(t-j T_{c}\right), t \in[0, T]$

where $N$ is the processing gain, $\left(p_{0}^{l}, p_{1}^{l}, \ldots p_{N-1}^{l}\right)$ is the signature sequence of $\pm 1^{\prime} s$ assigned to the $l$ th user, and $\varphi$ is the normalized chip waveform of duration $T_{c}$, with $N T_{c}=T$.

\section{A. Synchronous Case}

For the synchronous case (i.e., $\tau_{1}=\tau_{2}=\ldots=\tau_{l}=0$ ) of model (1), to demodulate the $i$ th symbols of the $L$ users, $\left\{b_{l}(i)\right\}_{l=1}^{L}$, it is sufficient to consider the received signal during the $i$ th signaling interval, i.e.,

$$
y(t)=\sum_{l=1}^{L} w_{l} b_{l}(i) x_{l}(t-i T)+z(t), t \in[i T,(i+1) T]
$$

Near the receiver, $y(t)$ is the received signal it is filtered by a chip-matched filter and then sampled at the chip rate. The resulting discrete-time signal sample corresponding to the $j$ th chip of the $i$ th symbol is given by

$$
\begin{gathered}
y_{j}(i)=\int_{i T+j T_{c}}^{i T+(j+1) T_{c}} y(t) \varphi\left(t-i T-j T_{c}\right) d t \\
(\text { or }) \\
=\sum_{l=1}^{L} w_{l} b_{l}(i) x_{j}^{l}+z_{l}(i) \\
\mathbf{y}(i)=\sum_{l=1}^{L} w_{l} b_{l}(i) \mathbf{x}_{l}+\mathbf{z}(i)
\end{gathered}
$$

where

$\mathbf{x}_{l}=\left[x_{0}^{l}, x_{1}^{l}, \ldots, x_{N-1}^{l}\right]^{T}=\frac{1}{\sqrt{N}}\left[p_{0}^{l}, p_{1}^{l}, \ldots, p_{N-1}^{l}\right]^{T}$ is the normalized signature sequence of the $l$ th user, and $\mathbf{z}(i)=\left[z_{0}(i), z_{1}(i), \ldots, z_{N-1}(i)\right]^{T}$ is the channel ambient noise sample vector at the $i$ th symbol interval. The term $\left\{z_{j}(i)\right\}$ is assumed as a sequence of independent and identically distributed (i.i.d.) random variables with a non Gaussian distribution. In this paper, the pdf of impulsive noise model is modeled as commonly used two-term Gaussian mixture model for the additive noise samples, $\left\{z_{j}(i)\right\}$, which has the form

$$
f=(1-\varepsilon) \varpi\left(0, v^{2}\right)+\varepsilon \varpi\left(0, \kappa v^{2}\right)
$$

with $v>0,0 \leq \varepsilon \leq 1$, and $\kappa \geq 1 . \varpi\left(0, v^{2}\right)$ term represents the nominal background noise and the $\varpi\left(0, \kappa v^{2}\right)$ term represents an impulsive component, with $\varepsilon$ representing the probability of 
occurrence of impulses. This model serves as an approximation to the more fundamental Middleton Class A noise model and also has been used extensively to model physical noise arising in radio and acoustic channels $[4,10,11]$.

\section{B. Extension to Asynchronous Case}

This algorithm is applicable for the users when they are symbol and chip asynchronous. After some algebra, asynchronous signal model given in (1) can be written as [5]

$$
\mathbf{y}_{m}(i)=\mathbf{H}_{m} \mathbf{W}_{m} \mathbf{b}_{m}(i)+\mathbf{z}_{m}(i)
$$

where

$$
\mathbf{H}_{m} \cong\left[\begin{array}{cccc}
\mathbf{H}(1) & \mathbf{H}(0) & \ldots & \mathbf{0} \\
: & \ldots & \ldots & : \\
: & \ldots & \ldots & : \\
\mathbf{0} & \ldots & \mathbf{H}(1) & \mathbf{H}(0)
\end{array}\right]_{N m \times L(m+1)}
$$

where $\mathbf{H}_{\mathrm{m}}$ denotes the $N M \times L(M+1)$ Hermitian cross correlation matrix of the composite waveforms associated with the symbols in $\mathbf{b}$ and the matrices $\mathbf{H}(0)$ and $\mathbf{H}(1)$ are defined as

$$
\left[\begin{array}{l}
\mathbf{H}(0) \\
\mathbf{H}(1)
\end{array}\right] \cong\left[\begin{array}{lll}
\mathbf{h}_{1} & \ldots & \mathbf{h}_{L}
\end{array}\right]
$$

with

$$
\mathbf{h}_{l} \cong\left(1-\pi_{l}\right)\left[\begin{array}{c}
\mathbf{0}_{m_{l}} \\
\mathbf{x}_{l} \\
\mathbf{0}_{N-m_{l}}
\end{array}\right]+\pi_{l}\left[\begin{array}{c}
\mathbf{0}_{m_{l}+1} \\
\mathbf{x}_{l} \\
\mathbf{0}_{N-m_{l}-1}
\end{array}\right]
$$

and $m_{l}=\left[\frac{\tau_{l}}{T_{c}}\right] \& \pi_{l}=\frac{\tau_{l}}{T_{c}}-m_{l}$.

that the Sylvester matrix $\mathbf{H}_{m}$ has full column rank. If the signature waveforms of all users as well as the relative delays are known to the receiver, then the Sylvester matrix $\mathbf{H}_{m}$ can be constructed.

Here, using PCA, principal components are calculated for received ym (i) as in eq(4)

The output matrix is is found as the covariance matrix 


$$
\begin{gathered}
y=\left[\begin{array}{cccc}
y(1) & y(2) & \ldots . & y(t) \\
y(0) & y(1) & \ldots & y(t-1) \\
y(-1) & y(0) & \ldots & y(t-2) \\
\vdots & \vdots & \ddots & y(1)
\end{array}\right] \\
\mathrm{C}=\operatorname{Covariance}(\mathrm{y}) \\
C=U \Sigma V^{T}
\end{gathered}
$$

\section{PRINCIPAl COMPONENT ANALYSIS AlgORITHM}

PCA is a technique which is generally used for reducing the dimensionality of multivariate data sets i.e. reducing the number of dimensions, without much loss of information .The covariance matrix of $\mathrm{n}$ random variables $\mathrm{x}$ is $\Sigma$, the principal components are defined as

$$
\mathrm{z}=\mathrm{Ax}
$$

Where $\mathrm{A}$ is $\mathrm{n}$ by $\mathrm{n}$ orthogonal matrix with rows of that are eigen vectors of covariance matrix as the rows of $\mathrm{z}$ is the vector of $\mathrm{n}$ principal components (PCs). The Eigen values of $\Sigma$ are proportional to the fraction of the total variance accounted for by the corresponding eigenvectors, so the PCs explaining most of the variance in the original variables can be identified. Regularly some of the original variables are correlated like a small group of the PCs describes a large space of the variance of the main data.

The data matrix $\mathrm{X}$ of size $\mathrm{m} \mathrm{x}$ is considered as received signal.

$$
X(t)=\left[x_{1}(t), x_{1}(t), x_{1}(t), \ldots \ldots x_{1}(t)\right]
$$

is the time ordered collection of a single matrix to which PCA can be applied. The means of the xi are removed and the covariance matrix computed by

$$
\sum=1 / n\left[X X^{T}\right]
$$

$\Sigma$ is an m x m square matrix, Eigen values, and corresponding eigenvectors will be calculated, First eigenvectors are calculated from the covariance matrix, second step is to arrange them by Eigen values in descending order. This gives you the components in order of significance. The lesser Eigen values are ignored there by reducing the data. Principal components are ordered eigenvectors of the covariance matrix. The PCs were obtained using

$$
\mathrm{z}_{\mathrm{j}}=\mathrm{a}_{\mathrm{j}} \mathrm{x} \quad \mathrm{j}=1,2, \ldots \ldots \mathrm{n}
$$

The PCs are a linear transformation with transformation coefficients given by the eigenvectors .

PCA is solved by using covariance matrix and singular value decomposition (SVD) methods. 
Let $\mathrm{X}$ be an arbitrary $\mathrm{n} \times \mathrm{m}$ matrix and $X^{T} X$. be a rank $\mathrm{r}$, square, symmetric $\mathrm{m} \times \mathrm{m}$ matrix. $\left\{\hat{v}_{1}, \hat{v}_{2}, \hat{v}_{3} \ldots \ldots \hat{v}_{r}\right\}$ is the set of orthonormal $\mathrm{m} \times 1$ eigenvectors with associated eigenvalues for $\left\{\lambda_{1}, \lambda_{2}, \lambda_{3}, \ldots \ldots \lambda_{r}\right\}$ the symmetric matrix $X^{T} X$.

$$
\left(X^{T} X\right) \hat{v}_{i}=\lambda_{i} \hat{v}_{i}
$$

$\sigma_{i}=\sqrt{\lambda_{i}}$ are positive, real and named as the singular values

$\left\{\hat{u}_{1}, \hat{u}_{2}, \hat{u}_{3} \ldots \ldots \hat{u}_{r}\right\}_{\text {is }}$ the group of $\mathrm{n} \times 1$ vectors defined by $\hat{u}_{i}=1 / \sigma_{i}\left[X \hat{v}_{i}\right]$

$\hat{u}_{i} \hat{u}_{j}=\left\{\begin{array}{ll}1 & i=j \\ 0 & i \neq j\end{array}\right.$ Eigenvectors are orthonormal.

$$
\left\|X \hat{v}_{i}\right\|=\sigma_{i}
$$

The scalar expansion of SVD is

$$
X \hat{v}_{i}=\sigma_{i} \hat{u}_{i}
$$

$\mathrm{X}$ multiplied by an eigenvector of $X^{T} X$.is equal to a scalar times another vector. The set of eigenvectors $\left\{\hat{v}_{1}, \hat{v}_{2}, \hat{v}_{3} \ldots \ldots \hat{v}_{r}\right\}$ and the set of vectors are $\left\{\hat{u}_{1}, \hat{u}_{2}, \hat{u}_{3} \ldots \ldots \hat{u}_{r}\right\}$ both orthonormal sets and bases in $r$ dimensional space.

$$
\Sigma=\left(\begin{array}{ccc}
\sigma_{1} & \ldots & 0 \\
\vdots & \ddots & \\
& \sigma_{\tilde{\gamma}} & \vdots \\
0 & \cdots & 0
\end{array}\right)
$$

$\sigma_{1} \geq \sigma_{2} \geq \sigma_{3} \ldots \geq \sigma_{\tilde{\gamma}}$ are the rank-ordered set of singular values. Likewise we construct accompanying orthogonal matrices,

$$
\begin{aligned}
& V=\left[\hat{v}_{1}, \hat{v}_{2}, \hat{v}_{3} \ldots \ldots \hat{v}_{r}\right] \\
& U=\left[\hat{u}_{1}, \hat{u}_{2}, \hat{u}_{3} \ldots \ldots \hat{u}_{r}\right]
\end{aligned}
$$

Matrix expansion of SVD

$$
X V=U \sum
$$


where each column of $\mathrm{V}$ and $\mathrm{U}$ perform the scalar version of the decomposition (Equation 3). Because $\mathrm{V}$ is orthogonal, we can multiply both sides by $\mathrm{V}-1=V^{T}$ to arrive at the final form of the decomposition.

$$
X V=U \sum V^{T}
$$

Implementation of PCA is as follows

The description of PCA using the covariance method is as follows:

The purpose is to transform one data set $\mathrm{X}$ of dimension $M$ to an another data set $\mathrm{Y}$ of smaller dimension $L$.

Step 1: Get some data and organize the data set.

Step 2: Subtract the mean:

For better performance of PCA, subtract the mean from each of the data dimensions.

The mean subtracted is equal to the average across each dimension.

Step 3: Calculate the covariance matrix.

Step 4: Calculate the eigenvectors and Eigen values of the covariance matrix.

Step 5: Choosing components and forming a feature vector:

The eigenvector containing the maximum Eigen value is considered as the principle component of the data set.

Feature Vector $=($ eigl, eig2, eig3, eig4,................, eign $)$.

Step 6: Deriving the new data set:

\section{EXPERIMENTAL RESULTS}

In simulations, first a synchronous system with 6 users is considered. The spreading sequence of each user with length 31 is considered (as shown in the following experiments). Figure 1(a-f) shows the bit error rate and signal to noise ratio comparisions for 6 different users which are estimated using corresponding principal componets. And the received users powers are all equal for the considered detectors (i.e., in the case of tight power control). The noise distribution parameters for are fixed as $\varepsilon=0.01 \& 0.1$ and $\kappa=100$, respectively. These plots demonstrate the performance gains achieved by the robust blind multiuser detector with PCA in impulsive noise. Next, an asynchronous system with 6 users and the noise distribution parameters $\quad \mathcal{E}=0.01 \& 0.1$ and $\kappa=100$ are considered. The delays of the 6 users are randomly generated. Fig 1( a-f) show the transmitter and estimated symbols for user 1.and Fig 2 show the BER for 6 different users with different Principal compon. Fig 3. Shows the details of transmitted and received symbols for of user one. Fig 4 gives the details of proposed method and subspace method. It is observed from these plots that the robust blind multiuser detector with the proposed PCA technique outperforms the traditional subspace method based linear MMSE detector in impulsive noise. The performance gain increases as the SNR increased. 


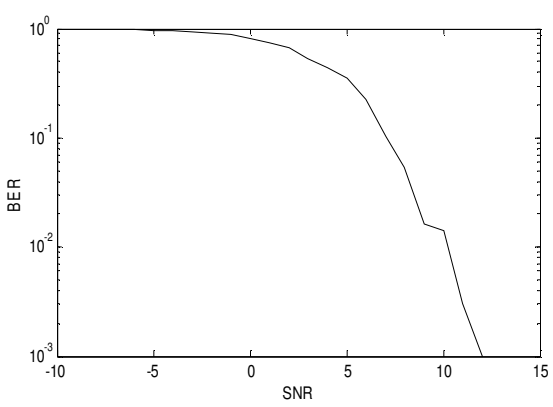

(a)

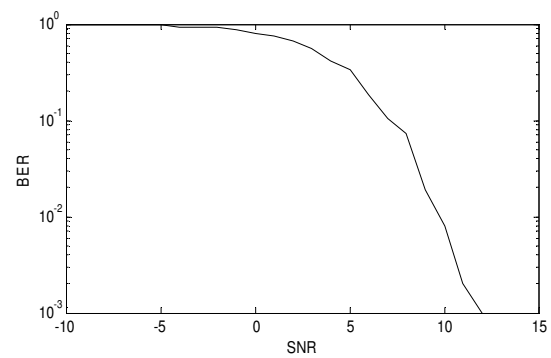

(c)

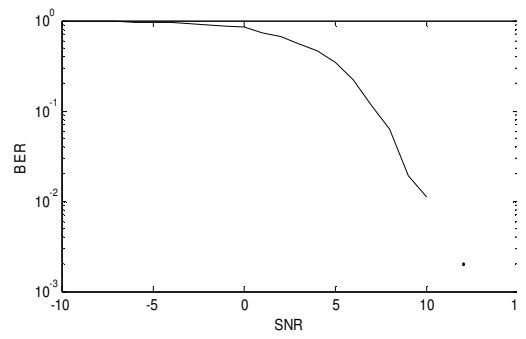

(e)

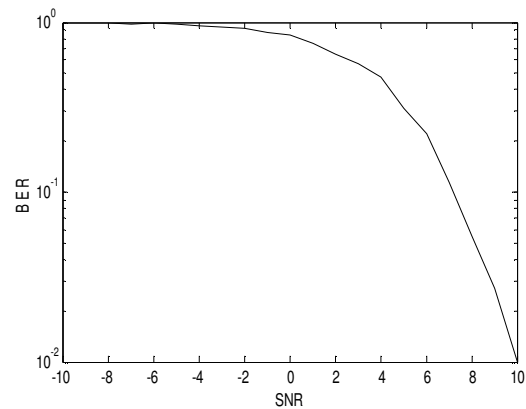

(b)

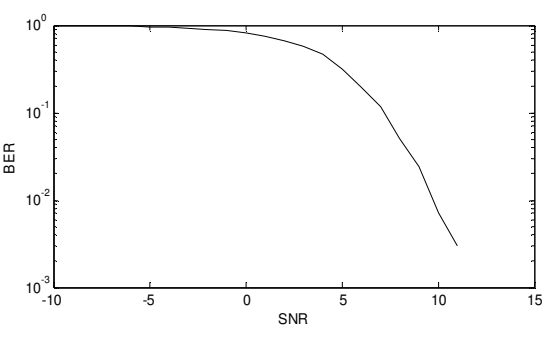

(d)

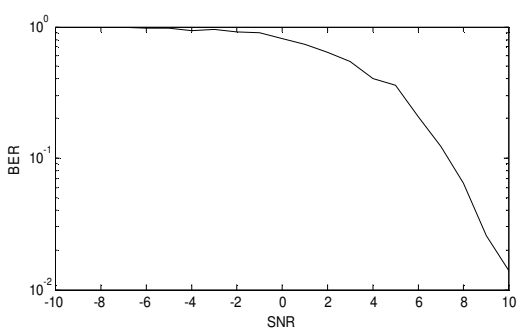

(f)

Fig 1. BER and SNR Plots for different users using first 6 Principal Components

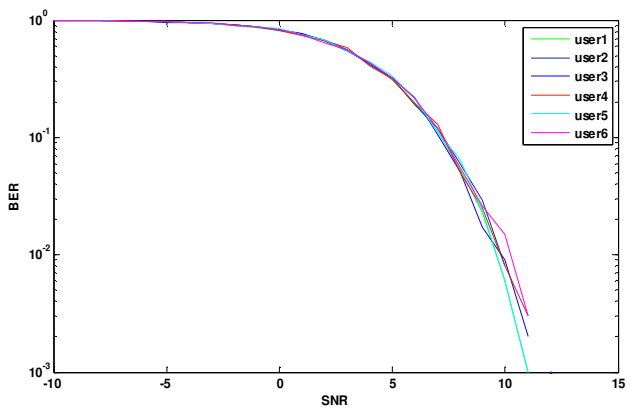

Fig 2 BER for 6 different users with different Principal components 

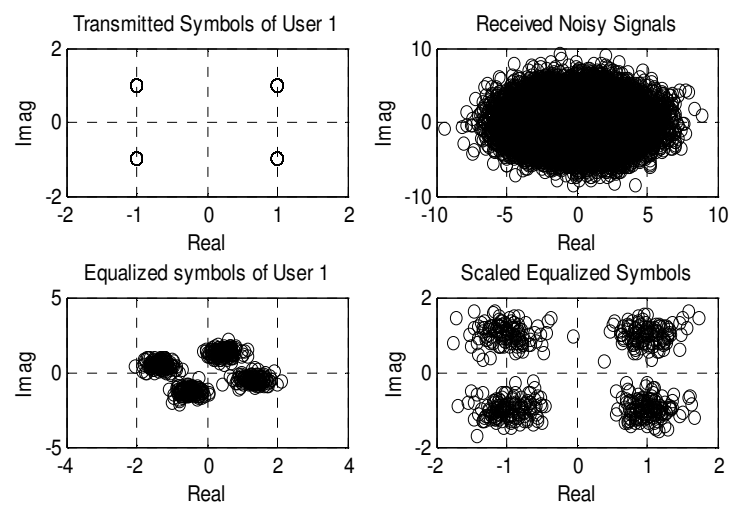

Fig 3. Transmitted and received symbols for user 1

\section{CONCLUSIONS}

In this paper, Multiuser detection has been done using principal components in asynchronous non-Gaussian channels is proposed and analyzed. Even the process of computing principal components is complex, simulation results show that the robust blind multiuser detector with the proposed PCA technique outperforms the traditional subspace method based linear MMSE detector in both synchronous and asynchronous CDMA non-Gaussian channels.

\section{REFERENCES}

[1] S. Verdu, Multiuser Detection, Cambridge University Press, Cambridge, 1998.

[2] E. Moulines, P. Duhamel, J. Cardoso, and S. Mayrargue, "Subspace Methods for the Blind Identification Multichannel FIR filters," IEEE Trans. on Signal Processing, vol. 43, pp. 516-525, Feb 1995.

[3] B.Yang, "Projection Approximation Subspace Tracking," IEEE Trans. on Signal Processing, vol. 43, pp. 95-107, Jan 1995.

[4] T.AnilKumar and K.DeerghaRao, “ Improved Robust Technique for Multiuser Detection in NonGuassian Channels", Circuits Systems and Signal Processing,vol 25,No 4,2006.

[5] X.Wang and H.V.Poor, "Robust Multiuser Detection in Non-Guassian Channels", IEEE Trans. On Signal Processing, vol. 47, pp. 289-305,Feb, 1999.

[6] G.W.Stewart, "An Updating Algorithm for Subspace Tracking”, IEEE Trans. On Signal Processing, vol. 40., pp. 1535-1541, June 1992

[7] U. Madhow and M.L. Honig. MMSE Interference Suppression for Direct-Sequence Spread-Spectrum CDMA. IEEE Trans. Communications, Vol. 42, No. 12, pp. 3188-3188, Dec. 1994.

[8] J.B. Schodorf and D.B. Williams. Array Processing Techniques for Multiuser Detection. IEEE Trans. Communications, Vol. 45, No. 11, pp. 1375 -1378, November 1997.

[9] M. Aminghafari, N. cheze, J.M Poggi,"Multivariate denoisious using wavlets and principal component Analysis," computational statistics \& Data Analsis, vol. 50 pp. 2381-2398, 2006

[10] ROBUST MIMO-CDMA DECORRELATING DETECTOR Hasan Abuhilal, Aykut Ho-canin and Huseyin Bilgekul Department of Electrical and Electronic Engineering Eastern Mediterranean University, Gazimagosa, via Mersin 10, Turkey

[11] R.Nirmaladevi,T.Anilkumar,K.K.Rao" Improved adaptive algorithm for blind multiuser detection in non gaussian noise" ISPPA2010Kuala Lumpur, Malaysia, 10-13 May, 2010. IEEE , ISBN 978-14244-7165-2, 270-273 